\title{
Cometary Silicates: Interstellar and Nebular Materials
}

\author{
Diane H. Wooden \\ NASA Ames Research Center, MS 245-3, Moffett Field, CA \\ 94035-1000, USA
}

\begin{abstract}
Evidence for interstellar material in comets is deduced from IR spectra, in situ measurements of Comet Halley, and chondritic porous interplanetary dust particles (CP IDPs). IR spectra of comets reveal the spectrally active minerals: amorphous carbon, amorphous silicates, and (in some comets) crystalline silicates. Evidence suggests amorphous silicates are of interstellar origin while crystalline silicates are of nebular origin.

$10 \mu \mathrm{m}$ spectra of comets and sub-micron amorphous silicate spherules in CP IDPs have shapes similar to absorption spectra through lines-of-sight in the ISM. Thermal emission models of cometary IR spectra require Fe-bearing amorphous silicates. Fe-bearing amorphous silicates may be Fe-bearing crystalline silicates formed in AGB outflows that are amorphized through $\mathrm{He}^{+}$ion bombardment in supernova shocks in the ISM.

Crystalline silicates in comets, as revealed by IR spectra, and their apparent absence in the ISM, argues for their nebular origin. The high temperatures $(>1000 \mathrm{~K})$ at which crystals form or are annealed occur in the inner nebula or in nebular shocks in the 5-10 AU region. Oxygen isotope studies of CP IDPs show only $1 \%$ by mass of the silicate crystals are of AGB origin. Together this suggests crystalline silicates in comets are probably primitive grains from the early solar nebula.
\end{abstract}

\section{Introduction}

Comets are frozen reservoirs of early solar nebula materials. During their perihelion passages, comets release volatile gases and dust particles into their comae. Cometary silicate mineralogy is deduced from IR spectra, in situ flyby mass spectrometry, and laboratory studies of cometary interplanetary dust particles. Analysis of cometary silicates suggests amorphous silicates are interstellar and crystalline silicates are formed or processed in the solar nebula (Wooden 2002).

\section{Silicate Mineralogy of Comets from IR Spectroscopy}

Long period and a few short period comets have broad silicate emission features. Some long period comets have crystalline silicate peaks: $11.2-11.4 \mu \mathrm{m}$ from Mg-rich crystalline olivine, and (in Comet Hale-Bopp) $9.2 \mu \mathrm{m}$ from Mg-rich crystalline ortho-pyroxene (Harker et al. 2002). Utilizing discrete mineral grains, thermal emission models of Comet Hale-Bopp show one-third of the sub-micron silicates are crystalline (Harker et al. 2002).

\section{Interstellar Fe-bearing Amorphous Silicates}

The $10 \mu \mathrm{m}$ absorption features seen along lines-of-sight through the interstellar medium are broad and attributed to amorphous silicates. In particular, Fe- 
bearing amorphous olivine fits the interstellar extinction curve ( $\mathrm{Li} \&$ Draine 2002). Fe-bearing amorphous olivine and pyroxene fit the absorption feature in the line-of-sight to the Galactic Center (Kemper, Vriend, \& Tielens 2004).

Aggregates of Fe-bearing amorphous silicates, Mg-rich silicate crystals, and carbonaceous materials constitute chondritic porous interplanetary dust particles (CP IDPs) of probable cometary origin (Fig. 1a) (Bradley et al. 1999b). The amorphous silicates in CP IDPs are $0.1-0.25 \mu \mathrm{m}$ silicate spherules with embedded nanophase Fe and FeS and are called GEMS (Glass with Embedded Metal and Sulfides) (Bradley et al. 1999a). Properties of GEMS have been attributed to ion bombardment in the interstellar medium (Bradley 1994). Laboratory experiments show $\mathrm{He}^{+}$ion bombardment of crystalline olivine destroys the crystalline structure and reduces the FeO bonds in the mineral to Fe, transforming the Fe-bearing crystal into a highly porous, $\mathrm{Mg}$-rich amorphous silicate with embedded nanophase Fe (Carrez et al. 2002). Absorption spectra of GEMS match the interstellar feature (Bradley et al. 1999a). Fe-bearing amorphous silicates in comets are probably from the interstellar medium.

\section{Nebular Mg-rich Crystalline Silicates}

In the interstellar medium, the fraction of crystalline silicates is very low $(<5 \%$ Li \& Draine 2002; <0.5\% Kemper et al. 2004), and is insufficient to account for the fraction deduced for comets. Mg-rich crystals (such as in Fig. 1c) may condense from nebular gases at $\sim 1400 \mathrm{~K}$ (Grossman 1972). Alternatively, Mgrich crystals may be Mg-rich amorphous silicates annealed into crystals $(\sim 900$ $1200 \mathrm{~K})$. Crystals annealed in the hot inner regions of the young $(<30,000 \mathrm{yr})$ solar nebula may be subsequently spread throughout the disk through turbulent dissipation (Bockelée-Morvan et al. 2002). Alternately, annealing may occur in nebular shocks in the 5-10 AU region (Harker \& Desch 2002). Laboratory experiments show Mg-rich amorphous olivine silicate smokes anneal into forsterite crystals (Fig. 1b) within a matrix of polycrystalline forsterite, crystalline and amorphous silica, and $\mathrm{MgO}$ (Fabian et al. 2000). Submicron sized particles with morphologies shown in Fig. 1b, c produce resonances of $\mathrm{Mg}$-rich crystalline olivine.

\section{Challenges}

- Oxygen isotopic nanoSIMS measurements of 1031 subgrains of 9 CP IDPs show that six grains ( $<1 \%$ by mass) have a very large anomalies and are stardust. One of the six pre-solar grains is a forsterite crystal, and two are GEMS (Messenger et al. 2003). If GEMS are interstellar, then why do most not show extreme anomalies? Future higher accuracy measurements of subgrains with modest anomalies will contribute to our understanding of the reservoir of materials that contributed to the solar system.

- Mg-rich crystals are annealed from Mg-rich amorphous silicates; annealing does not change the stoichiometry. However, amorphous silicates appear to have $\mathrm{Fe}$. What is the source of $\mathrm{Mg}$-rich amorphous silicates?

- CP IDPs contain discrete Mg-rich crystals (Fig. 1a; Bradley et al. 1999b) which do not appear morphologically similar to annealing products (Fig. 1b).

Acknowledgments. DHW thanks Drs. Don Brownlee and John Bradley. 

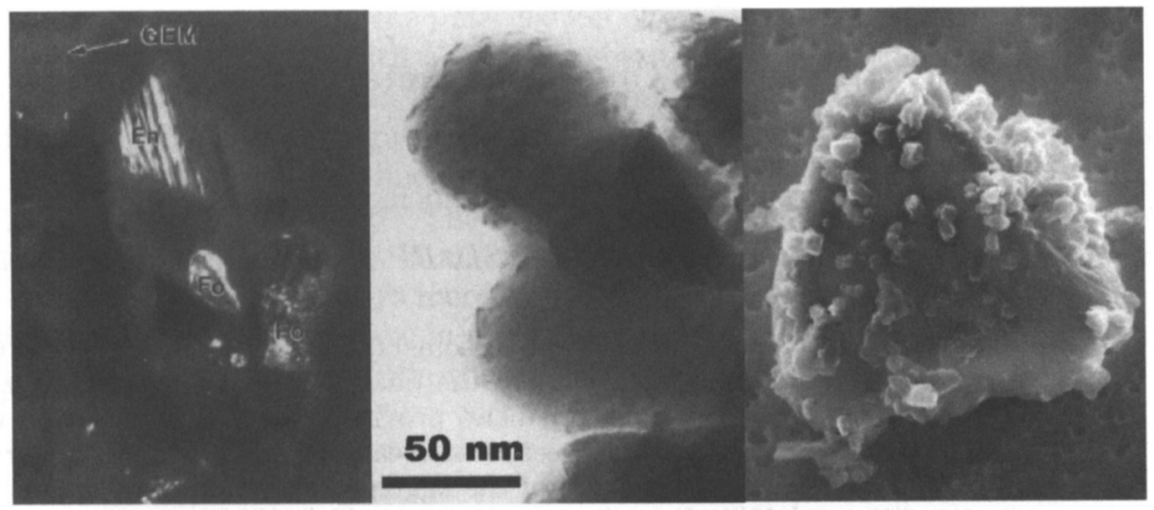

Figure 1. a. (Left) SEM view $(\sim 0.5 \mu \mathrm{m}$ wide) of a cometary CP IDP showing GEMS and Mg-rich silicate crystals (Fo and En). b. (Middle) Amorphous pure-Mg olivine smoke annealed at $1206 \mathrm{~K}$ for $1 \mathrm{hr}$ contains Forsterite crystals within an amorphous and polycrystalline matrix. c. (Right) $10 \mu \mathrm{m}$ Forsterite crystal IDP with GEMS attached. (Refs.: a, c: Fig. 4, 11, Bradley et al. 1999b; b: Fig. 11, Fabian et al. 2000.)

\section{References}

Bockelée-Morvan, D., Gautier, D., Hersant, F., Huré, J.-M., Robert, F. 2002, A\&A, 384,1107

Bradley, J. P. 1994, Science, 265, 925

Bradley, J. P., Keller, L. P., Gezo, J., Snow, T., Flynn, G. J., Brownlee, D. E., \& Bowey, J. 1999a, Lunar Planet. Sci., 30, Abs. No. 1835 The 10 and 18 Micrometer Silicate Features of GEMS: Comparison with Astronomical Silicates

Bradley, J. P., Keller, L. P., Snow, T. P., et al., Hanner, M. S., Flynn, G. J.,Gezo, J. C., Clemett, S. J., Brownlee, D. E., \& Bowey, J. E. 1999a, Science, 285, 1716 An infrared spectral match between GEMS and interstellar grains

Bradley, J. P., Snow, T. P., Brownlee, D. E., \& Hanner, M. S. 1999b in Solid Interstellar Matter: The ISO Revolution, Les Houches Workshop, ed. L. d'Hendecourt, C. Joblin, \& A. Jones (EDP Sciences and Springer-Verlag), 297

Carrez, P., Demyk, K., Cordier, P., Gengembre, L., Grimblot, J., d'Hendecourt, L., Jones, A. P., \& Lerouz, H. 2002, Meteorit. Planet Sci., 37, 1599

Fabian, D., Jäger, C., Henning, Th., Dorschner, J., \& Mutschke, H. 2000, A\&A, 364, 282

Grossman, L. 1972, Geochim. Cosmochim. Acta, 38, 47

Harker, D. E., \& Desch, S. 2002, ApJ, 565, L109

Harker, D. E., Wooden, D. H., Woodward, C. E., \& Lisse, C. M. 2002, ApJ, 580, 579

Kemper, F., Vriend, W. J., \& Tielens, A. G. G. M. 2004, ApJ, 609, 826

Li, A., \& Greenberg, J. M. 1997, A\&A, 323, 566

Li, A., \& Draine, B. T. 2002, ApJ, 564, 803

Wooden, D. H. 2002, Earth Moon Planets, 89, 247 